\title{
Localization of volatile isotopes on a cryotrap *
}

\author{
P.G. Thirolf ${ }^{a, b}$ M. Gross ${ }^{a, b}$ D. Habs ${ }^{a, b}$ A. Kohlhund ${ }^{a, b}$ F. Nebel ${ }^{c, b}$ J.B. Neumayr ${ }^{a, b}$ R. Stoepler ${ }^{a, c, b}$ \\ J. Szerypo a,b \\ ${ }^{a}$ Department f. Physik, LMU München, Garching, Germany \\ ${ }^{\mathrm{b}}$ Maier-Leibnitz-Labor, Garching, Germany \\ ${ }^{\mathrm{c}}$ Physik-Department, TU München, Garching, Germany
}

\begin{abstract}
Neutron-induced fission of uranium allows for the production of high-intensity neutron-rich radioactive ion beams. However, also large quantities of unwanted volatile radioactive species are produced that have to be hindered from contaminating the beamline and vacuum system of the facility. In the framework of radioprotection studies within the MAFF project at the FRM II in Garching with $10^{14}$ fiss/sec. [1], the performance of a cryotrap system has been studied, designed to localize gaseous radioactivity close to its origin. These studies provide important radioprotection information for the planned EURISOL facility with $10^{15}$ fission events/sec. Design considerations of a compact cryotrap operated with cold helium gas at a saturation temperature around $18 \mathrm{~K}$ will be presented. Activity distribution calculations of the fission source, the cryotrap and the subsequent vacuum system result in a prediction of the retention capability of the cryotrap system of $99.98 \%$. These design calculations have been experimentally verified with three cryotrap prototypes differing in cold surface area as well as in their internal helium gas flow characteristics. Retention capabilities have been measured with and without passive shielding of the external thermal load (300 K) using different tracer gases and an inclusive pressure-related diagnostics as well as mass-spectroscopic measurements.
\end{abstract}

Key words: radioprotection, volatile radioactivity, cryotrap

PACS: 28.41.Te, 28.52.Nh, 92.20.Td

\section{Introduction}

The ${ }^{235} \mathrm{U}$ fission source developed for the MAFF project consists of up to $2 \mathrm{~g}$ of ${ }^{235} \mathrm{U}$, dispersed in a porous graphite matrix surrounded by a Rhenium cylindre of about $8 \mathrm{~cm}$ length and $2 \mathrm{~cm}$ diameter, operating at a $\mathrm{HV}$ potential of $30 \mathrm{kV}$. Due to the large thermal neutron fission cross section $(582 \mathrm{~b})$ a rate of about $10^{14}$ fission events/sec. can be achieved with already $1.2 \mathrm{~g}$ of fissile material arranged in a compact target ion source unit in a total neutron flux at target position of $\Phi_{\mathrm{tot}}=9.7 \cdot 10^{13} \mathrm{n} \mathrm{cm}^{-2} \mathrm{~s}^{-1}$ and a thermalization level of $84 \%$. During the reactor cycle of 52 days, when the source is heated by the fission process and the fission products diffuse out of the Rhenium container, the target ion source has to be considered to be a $10 \mathrm{kCi}$ open source. It can be estimated that in a distance of $1 \mathrm{~m}$ from the

\footnotetext{
* Supported by the European Community under the FP6 EURISOL DS Project, Contract no. 515768 RIDS. The EC is not liable for any use that can be made of the information contained herein.

Email address: Peter.Thirolf@physik. uni-muenchen.de (P.G. Thirolf).
}

fission source a total dose of $3 \cdot 10^{8} \mu \mathrm{Sv} /$ hour resulting from non-volatile fission products will be produced. Only about $2 \%$ of the fission products will leave the source as ions, while about $30 \%$ of the fission products will be produced as volatile species. The MAFF cryosystem is designed to localize the dominant fraction of volatile and non-ionized radioactivity close to its origin at the fission source on the cold surface of a cryotrap so that only a fraction with a lower limit of $0.02 \%$ will escape to the outer beamline or will be deposited in the exhaust storage tanks of the vacuum system. This retainment capability of the cryotrap system has been derived from (time-independent) simulation calculations [2] of the radioactivity distribution along the MAFF beamline using the commercial MOVAK3D code [3].

Volatile Uranium fission products typically exhibit shortlived $\beta$ decay halflives in the order of minutes with only a few exceptions of longer-lived activity. It is the purpose of the cryopump to freeze out gaseous activity in order to localize it onto the cold surfaces until the $\beta$ decay will transform the dominant fraction of volatile activity into nonvolatile species. 
The cooling conditions needed to freeze out the radioactive gases can be clarified when looking at the vapour pressure curves of the different gaseous elements as a function of temperature, as shown in Fig. 1. For temperatures below about 20 Kelvin all relevant species including Nitrogen as the dominant component of the residual gas can be frozen out to partial pressures in the ultra-high vacuum regime. Therefore a temperature of $15 \mathrm{~K}$ was chosen as the operational temperature for the cryopump.

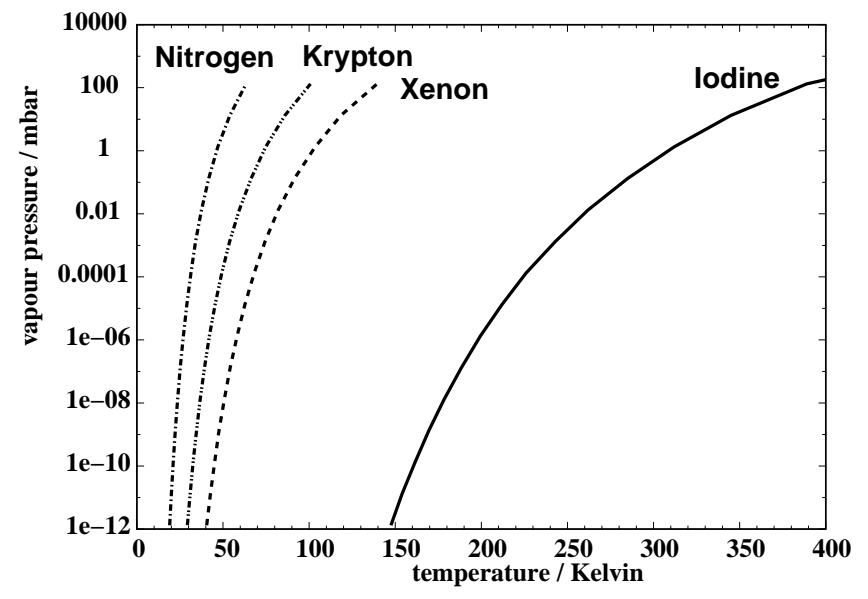

Fig. 1. Vapour pressure curves of volatile ion species.

\section{Cryopanel design and characterization}

The cryopanel designed to confine gaseous radioactivity near the MAFF fission source has to provide a homogeneous temperature distribution of about $15 \mathrm{~K}$ over a large area within a very limited space available in the MAFF beam tube. So a very compact design had to be found for the cryopanels. As a solution a double-walled tube design was chosen, where six spiral-type channel segments allow for a circulation of cold Helium gas. This can be seen in the upper part of Fig. 2, where the inner part of the double-walled tube with the spiral structure is shown that separates the six gas flow channels. A photograph of the cryopanel prototype (furtheron labeled 'large' panel) can be seen in the bottom part of Fig. 2.

The cryopanel has a length of $1 \mathrm{~m}$ with an inner open diameter of $157 \mathrm{~mm}$, which is limited by the diameter of the fission source that needs to be moved through the cryopanel assembly during the routinely exchange procedure. The wall thickness was chosen to be $2 \mathrm{~mm}$, which is the minimum value acceptable in view of the safety regulations to be obeyed. The gap for the cold Helium gas flow between the two tubes is $4 \mathrm{~mm}$. Due to its cryogenic specifications the cryopanel was manufactured from Al 6061T6. The assembly of the double-wall tube was performed by shrinking the (heated) outer tube onto the $\left(\mathrm{lN}_{2}\right.$-cooled) inner one. At the entrance side of the panel the cold gas is distributed via a manifold ring into three inlet channels, while the backflowing gas from the other 3 channels is funnelled into the outlet tube.

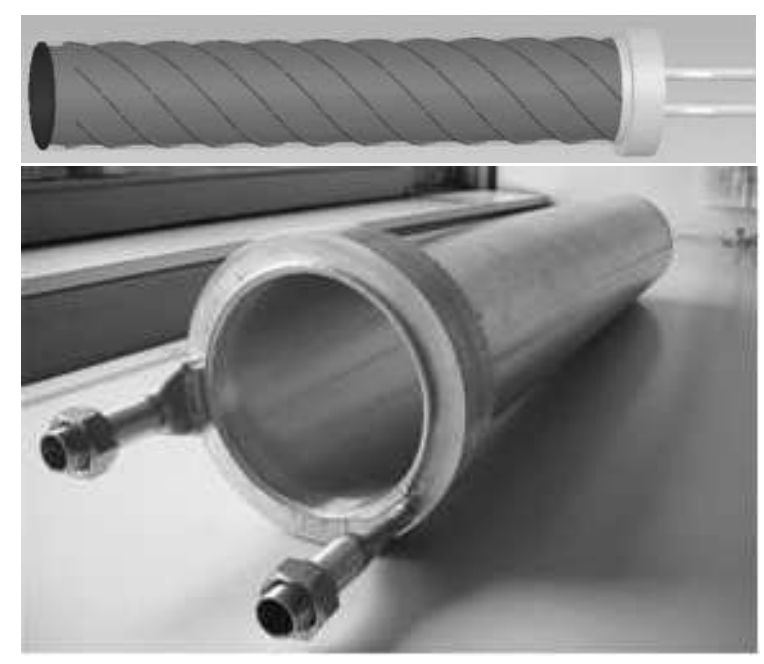

Fig. 2. Upper panel: design sketch of the inner part of the double-walled cryopanel showing the spiral structure separating the six gas flow channels. Bottom panel: photograph of the cryopanel prototype.

In order to compare the performance of this prototype with its rather complex mechanical design and gas flow pattern, two additional simplified cryopanel versions were built, one consisting of a $2 \times 1 \mathrm{~m}$ long U-shaped stainless-steel tube (labelled 'small' panel, see left part of Fig. 3) and a second one built from a copper spiral with similar length and diameter as the large panel (see right-hand part of Fig. 3).
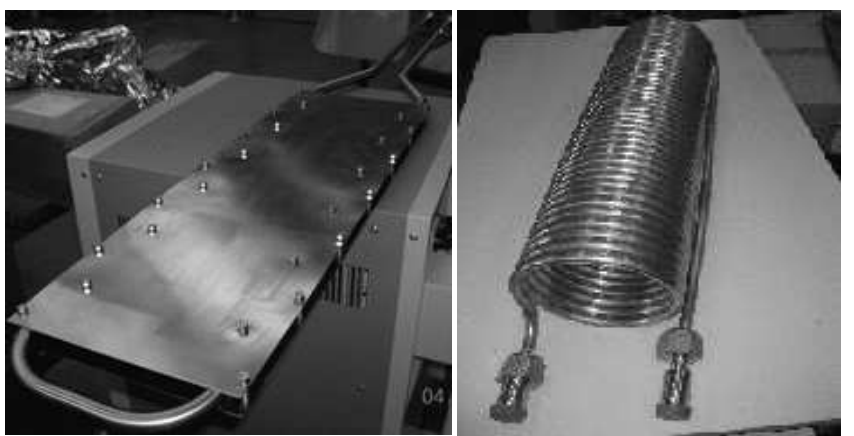

Fig. 3. Photographs of the two simplified cryotrap prototypes built for comparison with the 'large' panel. Left: 'small' panel built from a U-shaped steel tube with additional panel plates. Right: copper spiral panel.

In order to allow for systematic tests of the crypoanel prototype, a testbench was installed at the Maier-Leibnitz Laboratory in Garching, reproducing the dimensions of the inner part of the MAFF beamline. A sketch of this setup can be seen in Fig. 4.

The test-beamline is equipped with four vacuum pressure sensors and two mass spectrometers to both sides of the cryopanel. A calibrated needle valve coupled to a gas inlet system allows for rate-controlled test leakages. The cryopanel was equipped with six silicon diode thermal sensors mapping the temperature distribution along its surface. It was positioned inside the testbench and coupled via an isolated transfer line to a Helium liquifier, operated to produce cold Helium gas at about 10-15 K. The Helium 


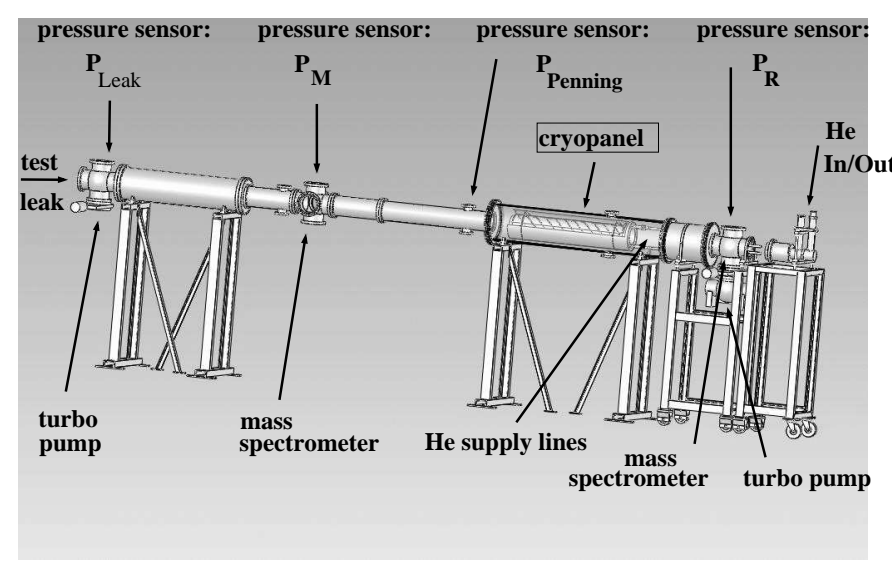

Fig. 4. Sketch of the cryopanel testbench at the MLL in Garching.

mass flow through the cryotrap amounted to $15 \mathrm{~g} / \mathrm{s}$. The cryopanel was centered via glass-epoxy (G10) rings with low thermal conductivity and small contact surface to the warm outside tube surface.

Pumping the testbench with two turbomolecular pumps at the ends of the beamline tube an ambient vacuum pressure of $1.5 \cdot 10^{-7}$ mbar was reached before cooling down the cryotrap. The basic observable studied in the subsequent tests was the so-called 'carry-over' factor, derived from the ratio of the pressure values measured before and behind the cryopanel, $\mathrm{P}_{R} / \mathrm{P}_{M}$. This value quantifies the amount of the non-localized gas load transmitted across the cryopanel, thus being complementary to the retention capability of the cryopanel.

\section{Results of cryotrap tests}

The retention capability of the cryotrap was tested by introducing a controlled test leakage ranging from $10^{-5} \mathrm{mbar}$ l/s up to 10 mbar l/s. The measurements shown in Fig. 5 were performed with nitrogen as test gas. The figure displays the 'carry-over' factor, i.e. the fraction of the nonlocalized amount of gas, as function of the vacuum pressure behind the cryotrap. Each data point corresponds to a certain test leakage rate. During these tests the gate valves between the turbo pumps and the beamline were closed.

The figure shows data measured with the 'large' cryopanel (labelled 'GP' in the plot) and with the 'small' panel (labelled 'SP') for different inlet temperatures of the cold helium gas (between $8 \mathrm{~K}$ and $30 \mathrm{~K}$ ). Since the active area of the small panel is about a factor of 18 smaller than the large one, a correspondingly reduced retention capability is visible for the small panel. For lower helium inlet temperatures an improved pumping capacity is found as expected (drastically visible for the two curves indicating the small test panel). The horizontal solid lines represent the results of Monte Carlo simulations of the radioactivity distribution along the MAFF beamline using the MOVAK3D code. Here for a single cryopanel (as used in our tests), a carry-over value of about $8 \cdot 10^{-4}$ is calculated (indicated in Fig. 5 as 'single panel'), while for the full setup of two cry- opanels placed to both sides of the MAFF fission source a value of $2 \cdot 10^{-4}$ is obtained (corresponding to $99.98 \%$ localization of volatile species onto the cryotrap). As can be seen from Fig. 5, the tests with the large cryotrap prototype confirm these calculated values, the non-localized gas load stays well below the design limit.

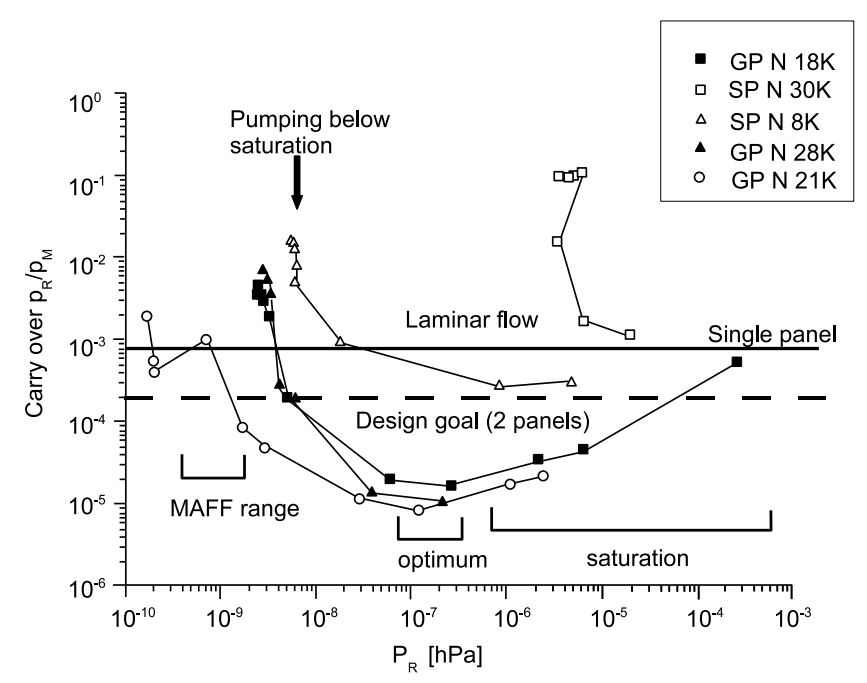

Fig. 5. Retention capability of the cryopanel (expressed by the 'carry-over'-factor reflecting the gas load transmitted across the cryotrap) as a function of the vacuum pressure behind the cryotrap.

During regular operation of the fission source, the production of volatile radioactivity will range around $10^{-5}$ mbar $1 / \mathrm{s}$, corresponding to the region labelled 'MAFF range' in Fig. 5. Here we find a pumping capacity of the cryotrap in agreement with the simulations for a single panel. Larger values of the test leakage rate exhibit a significantly improved localization capability with an optimum around a vacuum pressure of $10^{-7}-10^{-6}$ mbar behind the cryotrap, corresponding to a test leakage rate of ca. $10^{-3}$ $10^{-2} \mathrm{mbar} \mathrm{l} / \mathrm{s}$, since here the efficiency of the cryotrap is increased by the transition from molecular to laminar flow. However, this region already corresponds to a situation where during operation of the fission source the vacuum monitoring installations would have detected a leakage, initiating appropriate safety measures. In this failure scenario the increased retention efficiency of the cryotrap by more than an order of magnitude compared to the gas load regime during regular operation provides additional capacity for the containment of volatile radioactivity. Only if the leakage rate is further increased, saturation of the cryotrap will start to reduce the pumping capacity by emissivity changes due to condensate buildup on the cold surface.

In order to study the cryotrap performance for different volatile species, argon and krypton was used as test leakage gas besides nitrogen. Fig. 6 displays the vacuum pressure achieved behind the cryotrap as a function of the helium gas temperature for the large and small panel, respectively. The measurements were done at a fixed leakage rate of $5 \cdot 10^{-6} \mathrm{mbar} 1 / \mathrm{s}$. For comparison the dashed lines indicate the vapour pressure curves for the three gas species. 
While the data points within errors follow the trends of the vapour pressure curves down to about $25 \mathrm{~K}$, saturation sets in for lower temperatures caused by partial pressure contributions from surface outgassing as well as by emissivity changes on the cryotrap surface by condensate buildup.

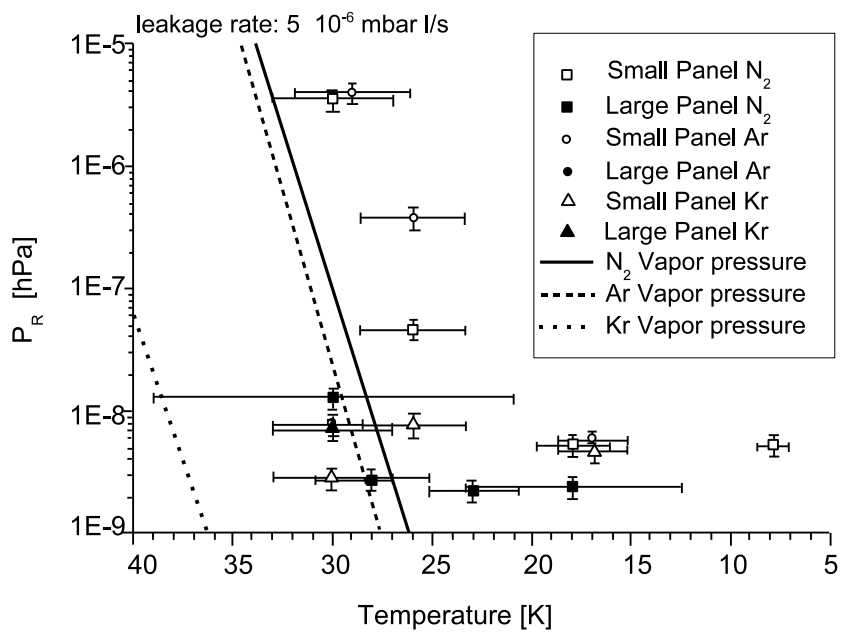

Fig. 6. Retention capability of the cryopanel (expressed by the vacuum pressure achieved behind the cryotrap) as a function of the helium gas temperature for different gases introduced as test leakage.

It should be mentioned that the cryotrap tests also revealed the technical challenges arising with the thermallyinduced mechanical stress to the cryotrap materials. Fig. 7 displays results of a finite-elements calculation using the Solid Works code, illustrating the mechanical deformation of the 'large' cryotrap for a static helium gas temperature of $15 \mathrm{~K}$ and a gas pressure of 3.5 bar. The figure shows an overlay of the warm, undeformed cryotrap tube (transparent outer layer in Fig. 7) and the cold cryopanel, where the greyscale values indicate the amount of thermally induced deformation. The resulting deformation is determined by the fixed bearing at the gas inlet side of the cryopanel and amounts to an axial contraction, which is gradually increasing from the connecting gas manifold at the back side to the front part of the cryotrap with its reversal region of the gas flow. Over the total tube length of $1000 \mathrm{~mm}$ an axial contraction of about $7 \mathrm{~mm}$ occurs, while the corresponding radial contraction amounts to about $1 \mathrm{~mm}$ (both to be seen in Fig. 7 as the difference between the outer transparent and inner grey-shaded cryotrap model). Due to the radially asymmetric mass distribution of the gas distribution manifold at the back side of the panel not only a length contraction, but also a deformation is induced, which is visible in Fig. 7 as a penetration between the warm and the cold cryotrap models.

As it turned out, after several cooling cycles the large cryopanel developed an internal leak between one of the incoming and outgoing helium channels, resulting in an inhomogeneous temperature distribution along the crytrap surface and a subsequent drastic loss in pumping capacity.

The reason may be attributed to the gradual release of internal mechanical stress within the aluminum tubes

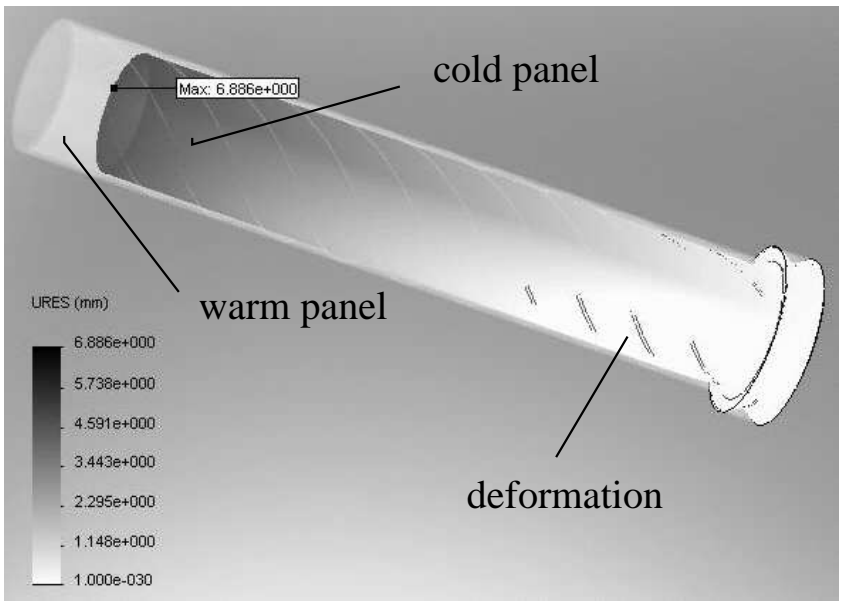

Fig. 7. Simulation of the thermal distortion of the cryotrap at $\mathrm{T}=15 \mathrm{~K}$.

forming the panel, resulting in a small gap developing between two of the spiral segments. This could be confirmed by neutron tomography applied to the cryotrap tube at the Garching neutron source FRM II. Thus a revised version of the cryotrap has to take special care of these thermal distortions.

In conclusion the performance of a He-cooled cryotrap was characterized at a temperature of $10-15 \mathrm{~K}$ and found in agreement with simulated values for the retention capability of volatile species. In this way it will be possible to localize $99.98 \%$ of unwanted volatile radioactivity from a fission source with $10^{14}$ fission events/sec. by using cryotraps to either side of the source in the beamtube. Effects of thermally-induced stress to the mechanical properties of the cryotrap design were encountered and included in a revision of the cryotrap design.

\section{References}

[1] D. Habs et al., The Munich Accelerator for Fission Fragments MAFF, Nucl. Instr. Meth. B204 (2003) 739

[2] F. Nebel et al., Radioactivity Distribution at MAFF, Eur. Phys. J. A 29 (2006) 369.

[3] G. Class, MOVAK3D, http://www.lv-soft.de (2004). 